This is the final peer-reviewed accepted manuscript of:

Alberto Martini, Marco Troncossi, Marco Carricato, Alessandro Rivola:

Static balancing of a parallel kinematics machine with LinearDelta architecture: theory, design and numerical investigation

In: Mechanism and Machine Theory, Volume 90, 2015, 128-141

The final published version is available online at:

https://doi.org/10.1016/i.mechmachtheory.2015.03.003

Rights / License:

The terms and conditions for the reuse of this version of the manuscript are specified in the publishing policy. For all terms of use and more information see the publisher's website.

This item was downloaded from IRIS Università di Bologna (https://cris.unibo.it/)

When citing, please refer to the published version. 


\title{
Static balancing of a Parallel Kinematics Machine with Linear-Delta architecture: theory, design and numerical investigation $^{1}$
}

\author{
Alberto Martini $^{\mathrm{a}, 2}$, Marco Troncossi ${ }^{\mathrm{a}, \mathrm{b}}$, Marco Carricato ${ }^{\mathrm{a}, \mathrm{b}}$, Alessandro Rivola ${ }^{\mathrm{a}, \mathrm{b}}$ \\ ${ }^{a}$ CIRI - Advanced Applications in Mechanical Engineering and Materials Technology, \\ University of Bologna, Viale del Risorgimento 2 - 40136 Bologna - Italy \\ ${ }^{b}$ DIN - Department of Engineering for Industry, University of Bologna, Viale del \\ Risorgimento 2 - 40136 Bologna - Italy
}

\begin{abstract}
This study deals with the compensation of gravity loads in parallel kinematics machines as a possible strategy for enhancing their working performance. In particular, the paper focuses on the static balancing of the Orthoglide 5-axis, a prototypal parallel robot with Linear-Delta architecture for machining operation. Gravity compensation is analytically carried out and a feasible design, based on tension springs and a simple additional linkage, is presented to implement the proposed strategy. Simulation results prove that the balancing device may provide an appreciable enhancement of the mechanism energy efficiency.
\end{abstract}

Keywords: static balancing, gravity compensation, Parallel Kinematics Machine, Linear-Delta

\section{INTRODUCTION}

A mechanism is statically balanced (or gravity compensated) if its total potential energy is constant for any admitted configuration. In such an instance, no motor actions are required to sustain the weight of the mechanism moving parts.

The compensation of gravity loads in industrial serial manipulators is a common practice and numerous balancing strategies have been investigated [1-5]. Conversely, static balancing of closed-loop mechanisms is still rare, even if this topic has been widely studied and several different approaches have been proposed, the majority of which are based on the introduction of counterweights and/or springs. While many techniques are suitable for planar closed-loop linkages [6-9], only relatively few solutions are available for spatial parallel manipulators, which is generally more challenging. The application of balancing counterweights normally entails a remarkable increment in the robot inertia

\footnotetext{
${ }^{1}$ A preliminary and partial version of this paper was presented at the ASME 12th Biennial Conference on Engineering Systems Design and Analysis (ESDA2014), June 25-27, 2014, Copenhagen, Denmark.

${ }^{2}$ Corresponding author. Tel.: +390543374440; Fax.: +390543374477.

E-mail addresses: alberto.martini6@unibo.it,marco.troncossi@unibo.it, marco.carricato@unibo.it, alessandro.rivola@unibo.it.
} 
$[10,11]$; when actuated prismatic joints are present, complex devices for making the counterweights translate are also required $[12,13]$. The use of compensation springs often requires the introduction of auxiliary linkages or even additional passive (not actuated) legs, which may interfere with the motion of other robot components $[10,11$, 14]. In some cases, an approximate compensation is preferred, in order to limit the complexity of the balanced robot $[15,16]$.

Parallel robots are frequently adopted in industrial applications for high-speed manipulation (e.g. pick and place tasks). In such an instance dynamic loads are predominant and the implementation of dynamic balancing techniques (rather than the mere compensation of gravity loads) is of primary interest. However, complete compensation of both shaking force and shaking moment (namely the resultant vector and the resultant moment of inertia actions) can be extremely difficult to achieve, and only the former is often compensated for in practice, thus achieving force balancing. Force balanced mechanisms are inherently statically balanced, since their overall center of mass (c.o.m.) is required to be stationary, and they were widely investigated [17]. Studies dealing with force balancing provide useful information concerning possible critical issues related to the introduction of balancing counterweights [18-21].

Static balancing is expected to bring about significant benefits to applications characterized by a heavy moving platform, long working periods in rest position and operations at low/moderate dynamics, since these features reasonably cause gravity loads to significantly affect the required actuator loads. Examples of parallel mechanisms characterized by the mentioned design features and functioning conditions are systems for robotically assisted surgery, flexible fixturing devices for assembly lines and Parallel Kinematics Machines (PKMs) for machining tasks. Indeed, studies concerning the implementation of gravity compensation strategies for improving the overall performance of such mechanical systems, particularly in terms of energy efficiency and safety, can be found in the literature [15, 22-25]. Nonetheless, a thorough investigation of the mechanism behavior in real working conditions is advisable, in order to verify the actual enhancement of the system operation as well as the presence of potential critical issues [20,21].

This paper studies the static balancing of a prototypal PKM characterized by a Linear-Delta architecture, namely the Orthoglide 5-axis [26]. The mechanism is a 5-DOF machine tool (simply referred to as Orthoglide hereafter) featuring a 2-DOF spherical wrist installed on the moving platform of a 3-DOF translational closed chain. The study focuses on the compensation of the gravity loads acting on the motors of the 3-DOF translational chain.

Gravity compensation is analytically achieved by using an approach based on constant-force generators $[13,27]$. Mechanical implementation is obtained by tension springs and a simple auxiliary linkage, and a practical design is presented that implements only commercial components. Since no balancing counterweights are used, no significant additional inertias are introduced.

The suitability of the proposed solution is investigated by testing numerical models of the unbalanced robot and its balanced variant. The dynamic operation is examined by simulating the execution of a number of reference trajectories and 
common machining tasks by means of inverse-dynamics analysis. A sensitivity analysis is performed for testing the robustness of the proposed design against the inaccuracies that could possibly affect its actual implementation (e.g. discrepancies between theoretical and actual values of the balancing parameters).

The structure of the paper is as follows. Section 2 describes the studied robot. Sections 3 and 4 present, respectively, the gravity-compensation strategy and the mechanical design of a feasible solution. Sections 5 and 6 report the numerical investigation. Section 7 draws some conclusions.

\section{THE ORTHOGLIDE 5-AXIS}

The Orthoglide 5-Axis is a prototypal 5-DOF hybrid PKM primarily conceived for milling operations [26]. A schematic is illustrated in Fig. 1. Three actuated legs, arranged according to a Linear-Delta architecture, drive a 3-DOF translating platform (Fig. 1a), on top of which an active 2-DOF spherical wrist controls the spindle orientation (Fig. 1b). Each leg (Fig. 1c) is connected to the fixed base by an actuated prismatic joint. The actuated-joint axes are mutually orthogonal, with the slider of one leg $\left(\operatorname{leg}_{2}\right)$ moving parallel to the gravity vector. $\operatorname{Leg}_{2}$ is therefore also referred to as vertical leg, whereas $\operatorname{leg}_{1}$ and $\operatorname{leg}_{3}$ are referred to as horizontal legs. Each horizontal leg features a planar parallelogram linkage made up of two links of length $L$ (whose value is the same for all parallelograms), and two links (roughly) five times shorter. Revolute joints connect the parallelogram shorter links to the slider and to the platform, with the rotational axes being parallel and lying on the parallelogram plane. The joint sequence is shown in Fig. 1c, where $P, R$ and $P a$ stand, respectively, for prismatic joint, revolute pair and parallelogram linkage. $\operatorname{Leg}_{2}$ features two distinct parallelograms. At the mechanism reference configuration, the $\operatorname{leg}_{2}$ parallelograms lie on mutually-orthogonal vertical planes, whereas the parallelograms of $\operatorname{leg}_{1}$ and $\operatorname{leg}_{3}$ lie on the same horizontal plane.

Each horizontal leg prevents 2 rotations of the platform, whereas the vertical leg constrains all rotational freedoms. As a result, the platform admits 3 translational DOFs, and the mechanism is overconstrained [28].

For the $j$-th leg $(j=1,2,3)$, points $O_{j}$ and $D_{j}$, respectively attached to the slider and the platform, and lying on the revolute joint axes, are defined as the median points between the parallelogram joint axes. A fixed coordinate system, $\Sigma=X Y Z$, is established as in Fig. 1a. It is centered in $O$, i.e. the intersection point of vectors $\mathbf{r}_{j}=\left(D_{j}-O_{j}\right), j=1,2,3$, at the home configuration, with axes $x, y$ and $z$ passing through points $O_{j}$. $P$ is a reference point on the platform that coincides with the intersection of the axes of the spherical wrist. The reference point $P$ also coincides with the origin of $\Sigma$ at the reference configuration. The translating platform is characterized by a cubic workspace (edges of $0.5 \mathrm{~m}$, parallel to the axes of $\Sigma$ ), whose center point, $C$, does not coincide with the origin 0 .

The spindle c.o.m., $G_{s p}$, is located very close to the intersection of the axes of the spherical wrist. Due to such a design feature, the static torques loading the wrist actuators are very small for any admissible spindle orientation, and may be reasonably neglected. For this reason, this study focuses on the compensation of the gravity loads acting on the translational kinematic chain, and static balancing of the spherical wrist is 
not performed. Accordingly, the variables describing the wrist pose, $\theta_{1}$ and $\theta_{2}$ (Fig. 1b), are neglected in the equations derived hereafter. Furthermore it may be proven that gravity compensation of the 3-DOF translational chain is not affected by the spindle orientation (see Sec. 3.3).

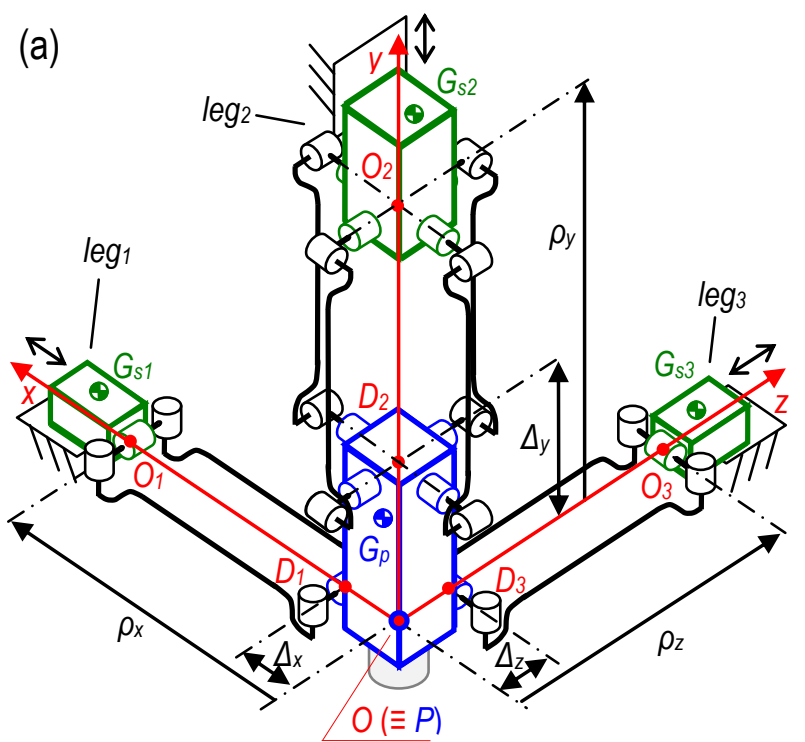

(b)

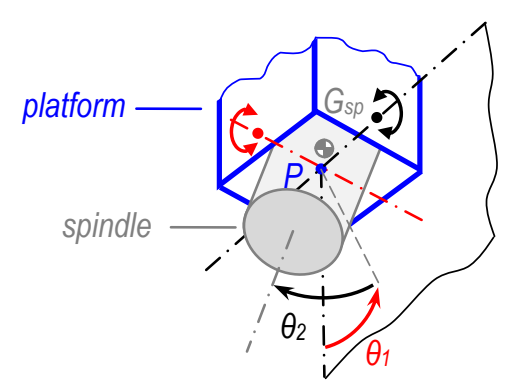

(c)

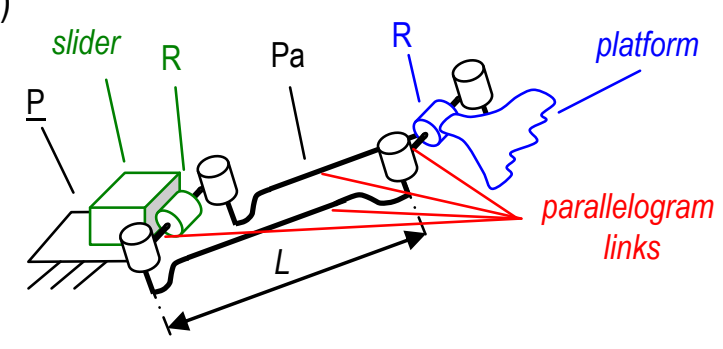

Fig. 1. Schematics of (a) the Orthoglide 5-axis at the reference configuration, (b) the spindle and (c) the kinematic chain of a horizontal leg. 
The solution of the inverse kinematics for a generic position vector $\left(p_{x}, p_{y}, p_{z}\right)$ of the platform reference point $P$ is given by [28]

$$
\left\{\begin{array}{l}
\rho_{x}=p_{x}+\Delta x+\sqrt{L^{2}-p_{y}^{2}-p_{z}^{2}} \\
\rho_{y}=p_{y}+\Delta y+\sqrt{L^{2}-p_{x}^{2}-p_{z}^{2}} \\
\rho_{z}=p_{z}+\Delta z+\sqrt{L^{2}-p_{x}^{2}-p_{y}^{2}}
\end{array}\right.
$$

where the scalar parameters $\rho_{x}, \rho_{y}, \rho_{z}$ describe the position of the slider points $O_{j}$ on the corresponding axis of $\Sigma$, and the scalar constants $\Delta x, \Delta y, \Delta z$ are the offsets of points $D_{j}$ with respect to the reference point $P$.

\section{THE GRAVITY-BALANCED ORTHOGLIDE 5-AXIS}

The approach adopted for the static balancing of the Orthoglide is based on the concept of constant-force generators. Firstly, a set of constant forces (more precisely, constant-magnitude forces with fixed orientation), exerted by the legs on the moving platform, is determined by imposing the neutral equilibrium of the platform. Then, suitable balancing devices (typically comprising springs and/or counterweights) are introduced in each leg so that the constant forces computed at the previous step may be actually generated. The parameters characterizing the balancing devices are determined by imposing the invariance of the total potential energy of the leg, which is given by the contribution of all conservative forces, i.e. gravity loads, spring forces and the required constant force.

Carricato and Gosselin [13] derived the necessary and sufficient conditions for the neutral equilibrium of a rigid body arbitrarily moving in space and acted upon by its own weight and by a number of constant forces. Such conditions assume a particularly simple form for the moving platform of the Orthoglide, which has a purely translational motion. In this case, a sufficient condition for static balancing is the equilibrium of the platform own weight $\left(\mathbf{F}_{p}\right)$ and the constant forces applied by the legs $\left(\mathbf{f}_{j}\right)$, i.e.

$$
\mathbf{F}_{p}+\sum_{j=1}^{3} \mathbf{f}_{j}=0
$$

A mass distribution which is analytically equivalent to the real one from the static-balancing point of view is adopted. Indeed, only four lumped mass parameters ${ }^{3}$ may be used to describe the Orthoglide gravity loads: $m_{p}$, located at point $G_{p}$ of the platform, which accounts for the total mass of the platform, including the spindle, the spherical wrist and half the mass of each parallelogram; and $m_{s j}, j=1,2,3$, located at the slider point $G_{s j}$, which accounts for the total mass of the slider and the remaining mass of the parallelogram belonging to the corresponding leg.

The sliders of the horizontal legs translate orthogonally to the gravity vector, so that the potential energy of masses $m_{s 1}$ and $m_{s 3}$, is constant. Hence, the static balancing of the Orthoglide may be achieved by transforming its vertical leg into a constant-force

\footnotetext{
${ }^{3}$ For each parallelogram, the masses of the shorter links are added, respectively, to the platform and to the slider, since their c.o.m. are fixed with respect to such components. Half the mass of each longer link is added to both the platform and the slider, since the c.o.m. is located at the link median point.
} 
generator that exerts a force equal and opposite to the platform weight (i.e. $\mathbf{f}_{2}=-\mathbf{F}_{p}$ ), while leaving the horizontal legs unaltered, i.e. $\mathbf{f}_{1}=\mathbf{f}_{3}=\mathbf{0}$.

In such an instance, a condition of invariant total potential energy can be achieved by adding two convenient devices to $\operatorname{leg}_{2}$ :

- a balancing device that compensates for the potential-energy variations induced by the relative motion between the platform and the slider of the vertical leg, referred to as distal balancing device;

- a balancing device that compensates for the relative motion between the slider of the vertical leg and the fixed base, referred to as proximal balancing device.

\subsection{Distal balancing device}

A zero-free-length tension spring may be adopted as the distal balancing device (Fig. 2). The spring, with stiffness $K_{D}$, acts between the slider and the platform, with $A$ and $B$ being its anchor points on the slider and the platform, respectively. Points $A$ and $B$ are defined by constant vectors $\mathbf{a}=\left(a_{x}, a_{y}, a_{z}\right)$ and $\mathbf{b}=\left(b_{x}, b_{y}, b_{z}\right)$ from points $O_{2}$ and $D_{2}$ respectively. $\mathbf{a}$ and $\mathbf{b}$ have equal components along the $X$-axis and $Z$-axis, i.e. $b_{x}=a_{x}$ and $b_{z}=a_{z}$.

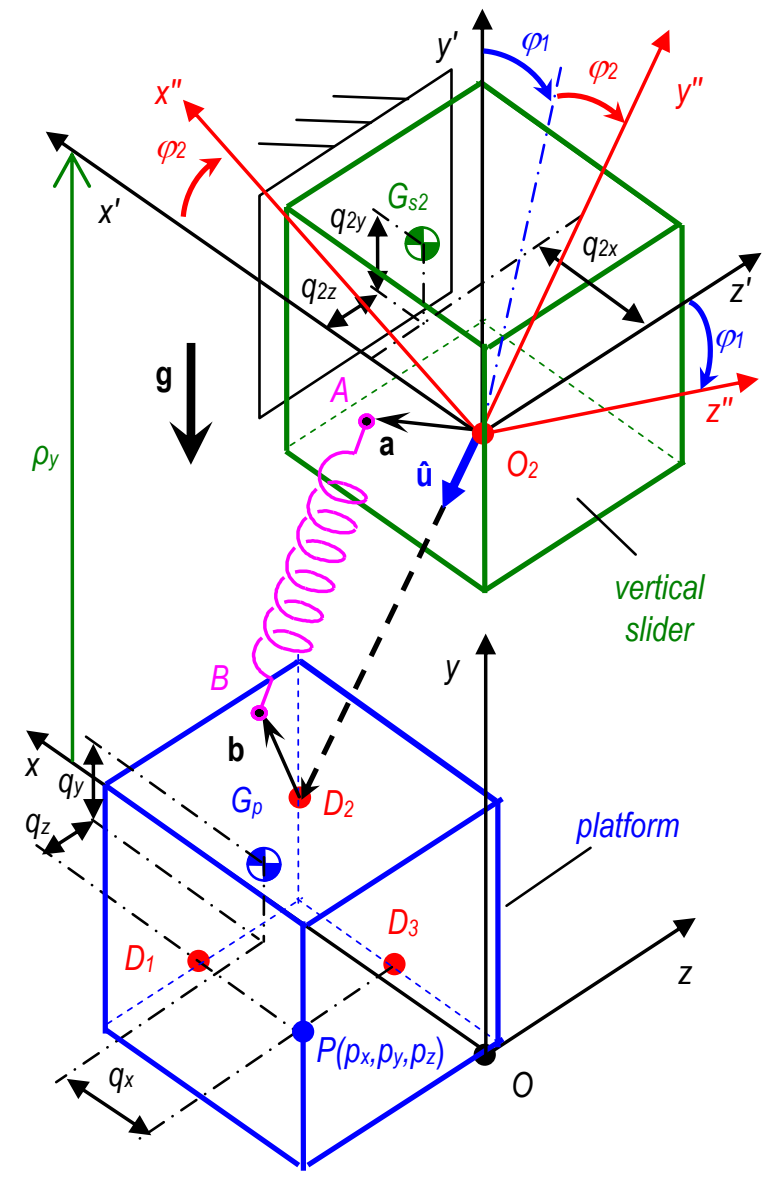

Fig. 2. Schematic of the platform and the $\operatorname{leg}_{2}$ slider for a generic configuration, with coordinate systems $\Sigma, \Sigma^{\prime}$ and $\Sigma^{\prime \prime}$. 
For balancing purposes, the configuration of the Orthoglide can be described by a set of independent variables related to the configuration of $\operatorname{leg}_{2}$, i.e. $\rho_{y}, \varphi_{1}$ and $\varphi_{2}$. Two floating coordinate systems, $\Sigma^{\prime}=X^{\prime} Y^{\prime} Z^{\prime}$ and $\Sigma^{\prime \prime}=X^{\prime \prime} Y^{\prime \prime} Z^{\prime \prime}$, both centered in $O_{2}$, are established as shown in Fig. 2. $\Sigma^{\prime}$ is attached to the slider of $\operatorname{leg}_{2}$ and oriented like $\Sigma$. $\Sigma^{\prime \prime}$ is arranged so that $Y^{\prime \prime}$ is aligned with $\mathbf{r}_{2}$, with opposite direction, and $Z^{\prime \prime}$ is perpendicular to the $X^{\prime} Y^{\prime \prime}$-plane. Euler angles $\varphi_{1}$ and $\varphi_{2}$ define the orientation of $\Sigma^{\prime \prime}$ with respect to $\Sigma^{\prime}$ and describe the relative motion between the platform and the slider: $\varphi_{1}$ is the rotation around the $X^{\prime}$-axis, whereas $\varphi_{2}$ is the rotation around the $Z^{\prime \prime}$-axis, i.e. the new $Z^{\prime}$-axis after rotation $\varphi_{1}$. The rotation matrix that gives the components in $\Sigma^{\prime}$ of a vector described in $\Sigma^{\prime \prime}$ is:

$$
\mathbf{R}\left(\varphi_{1}, \varphi_{2}\right)=\left(\begin{array}{ccc}
c_{2} & -s_{2} & 0 \\
c_{1} s_{2} & c_{1} c_{2} & -s_{1} \\
s_{1} s_{2} & s_{1} c_{2} & c_{1}
\end{array}\right)
$$

where $s_{i}$ and $c_{i}$ stand for sine and cosine of the angle $\varphi_{i}(i=1,2)$, respectively. The unit vector $\hat{\mathbf{u}}=(0,-1,0)$ describes the orientation of vector $\mathbf{r}_{2}$ in $\Sigma^{\prime \prime}$. The position vectors relevant for potential-energy computation are:

$$
\begin{aligned}
& \mathbf{r}_{O_{2}}=\left(0, \rho_{y}, 0\right) \\
& \mathbf{r}_{D_{2}}=\mathbf{r}_{O_{2}}+L \mathbf{R} \hat{\mathbf{u}}=\left(L u_{x}, \rho_{y}+L u_{y}, L u_{z}\right) \\
& \mathbf{r}_{P}=\left(L u_{x}, \rho_{y}+L u_{y}-\Delta y, L u_{z}\right) \\
& \mathbf{r}_{G p}=\left(L u_{x}+q_{x}, \rho_{y}+L u_{y}-\Delta y+q_{y}, L u_{z}+q_{z}\right) \\
& \mathbf{r}_{G s 2}=\left(q_{2 x}, \rho_{y}+q_{2 y}, q_{2 z}\right) \\
& \mathbf{r}_{A}=\left(a_{x}, \rho_{y}+a_{y}, a_{z}\right) \\
& \mathbf{r}_{B}=\left(L u_{x}+a_{x}, \rho_{y}+L u_{y}+b_{y}, L u_{z}+a_{z}\right) \\
& \mathbf{r}_{A B}=\left(\mathbf{r}_{B}-\mathbf{r}_{A}\right)=\left(L u_{x}, L u_{y}-\delta_{y}, L u_{z}\right)
\end{aligned}
$$

where $\delta_{y}=a_{y}-b_{y}$, and $\left(u_{x}, u_{y}, u_{z}\right)=\mathbf{R} \hat{\mathbf{u}}=\left(s_{2},-c_{1} c_{2},-s_{1} c_{2}\right)$ are the components of $\hat{\mathbf{u}}$ expressed in $\Sigma^{\prime}$.

If friction is neglected, only the gravitational and the spring forces act on the platform and $\mathrm{leg}_{2}$. Their contributions to the total potential energy of the Orthoglide are, respectively:

$$
\begin{aligned}
& V_{g D}=-m_{s 2} \mathbf{g} \cdot \mathbf{r}_{G s 2}-m_{p} \mathbf{g} \cdot \mathbf{r}_{G p}= \\
& =g\left[\left(m_{s 2}+m_{p}\right) \rho_{y}-m_{p} L c_{1} c_{2}+\psi\right] \\
& V_{e D}=\frac{1}{2} K_{D}\left(r_{A B}\right)^{2}=K_{D} \delta_{y} L c_{1} c_{2}+\lambda
\end{aligned}
$$

where $\psi=\left[m_{s 2} q_{2 y}+m_{p}\left(q_{y}-\Delta_{y}\right)\right]$ and $\lambda=\left[K_{D}\left(\delta_{y}{ }^{2}+L^{2}\right) / 2\right]$ are constants, and $\mathbf{g}=(0,-g, 0)$ is the gravity vector. 


\subsection{Proximal balancing device}

A schematic of the proximal balancing device is shown in Fig. 3. The component referred to as spring support is connected to the base by means of two parallel links, i.e. $l_{i n k_{1}}$ and $l i n k_{2}$, and to the slider by means of link $k_{3}$. All these links have length $H$. The planar mechanism made up by link ${ }_{1}$, link $k_{2}$, link $k_{3}$, the spring support, the vertical slider and the base has 1 DOF, which can be described by the variable $\rho_{y}$. A zero-free-length tension spring is connected to the spring support and to the slider, at points $N_{2}$ and $N_{1}$, respectively. $N_{1}$ and $N_{2}$ are defined by constant vectors $\mathbf{n}_{1}=\left(n_{1 x}, n_{1 y}\right)$ and $\mathbf{n}_{2}=\left(n_{2 x}, n_{2 y}\right)$, which have equal components along the $X$-axis, i.e. $n_{1 x}=n_{2 x}$.

The linkage presented here is an evolution a device commonly used to achieve the static balancing of 2-DOF planar open chains, a device that consists of two parallelograms connected in series and two springs [29]. Since, in the case at hand, the slider is constrained by a prismatic joint, one link can be removed from one parallelogram, and a single spring may be used. The proposed solution achieves gravity compensation with a rather limited increment of dimensions. Moreover, by connecting the balancing spring directly to the slider and to the spring support, only traction/compression loads act on each link $k_{k}$. Hence, these components may be designed as slender elements, thus entailing little inertia increment.

Similarly to the procedure adopted for the legs, an equivalent mass distribution of link $_{k}(k=1,2,3)$ is considered for gravity-compensation purpose, by concentrating half the mass of link $k_{k}$ in the center point of each one of its pivots. Two lumped masses are, thus, adopted to describe the gravity loads of the balancing device: $m_{w}$, equal to half the mass of $l i n k_{3}$ and located at the pivot $G_{w}$ of $l i n k_{3}$ on the vertical slider; and $m_{t}$, located in the c.o.m. of the spring support, $G_{t}$, given by both the total mass of the spring support and the contributions of link $k_{1}$, link $_{2}$ and link $k_{3}$.

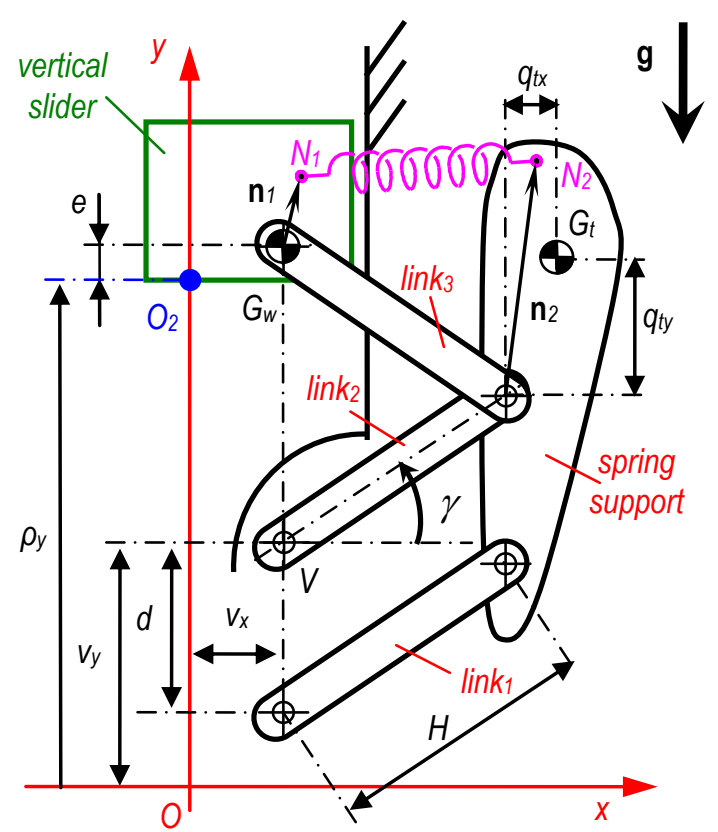

Fig. 3. Schematic of the proximal balancing device. 
The position vectors relevant for potential-energy calculation are:

$$
\begin{aligned}
& \mathbf{r}_{G t}=\left(H c_{\gamma}+v_{x}+q_{t x}, H s_{\gamma}+v_{y}+q_{t y}\right) \\
& \mathbf{r}_{G w}=\left(v_{x}, \rho_{y}+e\right) \\
& \mathbf{r}_{N_{1}}=\left(v_{x}+n_{1 x}, 2 H s_{\gamma}+v_{y}+n_{1 y}\right) \\
& \mathbf{r}_{N_{2}}=\left(H c_{\gamma}+v_{x}+n_{2 x}, H s_{\gamma}+v_{y}+n_{2 y}\right) \\
& \mathbf{r}_{N_{2} N_{1}}=\left(r_{N_{1}}-r_{N_{2}}\right)=\left(-H c_{\gamma}, H s_{\gamma}-\varepsilon_{y}\right)
\end{aligned}
$$

where $\varepsilon_{y}=n_{2 y}-n_{1 y}$ is a constant, and $s_{\gamma}$ and $c_{\gamma}$ stand, respectively, for sine and cosine of the auxiliary angle $\gamma$, which is related to $\rho_{y}$ by the equation

$$
H s_{\gamma}=\frac{\rho_{y}+e-v_{y}}{2}
$$

The contributions to the system potential energy, given by gravity loads and spring actions are, respectively:

$$
\begin{gathered}
V_{g P}=-m_{t} \mathbf{g} \cdot \mathbf{r}_{G t}-m_{w} \mathbf{g} \cdot \mathbf{r}_{G w}=g\left[\left(m_{w}+\frac{m_{t}}{2}\right) \rho_{y}+\mu_{1}\right] \\
V_{e P}=\frac{1}{2} K_{P}\left(r_{N_{2} N_{1}}\right)^{2}=-\frac{1}{2} K_{P} \varepsilon_{y} \rho_{y}+\mu_{2}
\end{gathered}
$$

where the terms $\mu_{1}=\left[m_{t}\left(e+v_{y}+2 q_{t y}\right) / 2+m_{w} e\right]$ and $\mu_{2}=\left\{K_{P}\left[\varepsilon_{y}{ }^{2}+H_{y}{ }^{2}-\varepsilon_{y}\left(e-v_{y}\right)\right] / 2\right\}$ are constants.

\subsection{Computation of the parameters of the balancing devices}

The total potential energy of the balanced Orthoglide is

$$
V_{T O T}=V_{g D}+V_{e D}+V_{g P}+V_{e P}+V_{g H}
$$

where $V_{g H}$ represents the gravitational potential energy related to masses $m_{s 1}$ and $m_{s 3}$, which remains constant during motion.

In order to obtain gravity compensation, $V_{\text {TOT }}$ must be constant for any set of parameters describing the mechanism configuration. Accordingly, the following conditions

$$
\left\{\begin{array}{l}
\frac{\partial V_{T O T}}{\partial \varphi_{1}}=\left(m_{p} g_{y}-K_{D} \delta_{y}\right) L s_{1} c_{2}=0 \\
\frac{\partial V_{T O T}}{\partial \varphi_{2}}=\left(m_{p} g_{y}-K_{D} \delta_{y}\right) L c_{1} s_{2}=0 \\
\frac{\partial V_{T O T}}{\partial \rho_{y}}=\left(m_{p}+m_{s 2}+m_{w}+\frac{m_{t}}{2}\right) g-\frac{1}{2} K_{p} \varepsilon_{y}=0
\end{array}\right.
$$

must be satisfied for any admissible value of $\varphi_{1}, \varphi_{2}$ and $\rho_{y}$, and thus: 


$$
\left\{\begin{array}{c}
K_{D} \delta_{y}=m_{p} g_{y} \\
K_{P} \varepsilon_{y}=\left(2 m_{p}+2 m_{s 2}+2 m_{w}+m_{t}\right) g
\end{array}\right.
$$

Since the system formed by Eqs. (15) and (16) has four unknowns (namely the constants $K_{D}, \delta_{y}, K_{p}$ and $\varepsilon_{y}$ ), a solution can be found by arbitrarily choosing two parameters. In particular, by recalling that

$$
\delta_{y}=a_{y}-b_{y}, \quad \varepsilon_{y}=n_{2 y}-n_{1 y}
$$

the springs anchor points (which affect $\delta_{y}$ and $\varepsilon_{y}{ }^{4}$ ) may be arbitrarily located on the platform, on the vertical slider and on the spring support, in order to meet the design specifications and constraints of the Orthoglide, whereas the spring rates may be computed as

$$
\begin{gathered}
K_{D}=\frac{m_{p} g}{\delta_{y}} \\
K_{P}=\frac{2\left(m_{p}+m_{s 2}+m_{w}+\frac{m_{t}}{2}\right) g}{\varepsilon_{y}}
\end{gathered}
$$

$K_{D}$ defines the stiffness of the tension spring comprised in the distal device, which compensates for energy variations related to variables $\varphi_{1}$ and $\varphi_{2}$, whereas $K_{p}$ defines the stiffness of the tension spring comprised in the proximal device, which compensates for energy variations related to variable $\rho_{y}$. Since the parameters $\delta_{y}$, and $\varepsilon_{y}$ are constant values, the spring rates $K_{D}$ and $K_{p}$ are constant as well. As a result, the gravity compensation of the Orthoglide can be achieved by means of tension springs characterized by constant stiffness parameters, $K_{D}$ and $K_{P}$, and connected to the platform, the vertical slider and the additional spring support at fixed anchor points. No counterweights are needed, and the overall inertia of the system is not significantly affected.

It is worth observing that the exact position of the platform c.o.m. $G_{p}$ may depend on the orientation of the spindle, since the mass of the spindle is taken into account for determining the platform mass properties and the spindle c.o.m. may not exactly coincide with the wrist center (Sec. 2). However, it may be shown that the position of $G_{p}$ does not affect the equilibrium of the platform, due to its translational motion, and thus the exact gravity compensation of the 3-DOF parallel chain is granted for any spindle pose.

\section{MECHANICAL DESIGN OF THE BALANCING DEVICES}

The introduction of cables and pulleys for transmitting the spring forces to the spring anchor points theoretically determined in Section 3 is advisable [14]. Indeed their use brings about the following advantages:

\footnotetext{
${ }^{4}$ The components of vectors $\mathbf{a}, \mathbf{b}, \mathbf{n}_{1}$ and $\mathbf{n}_{2}$ along the $X$-axis and $Z$-axis have no effect on balancing, since $\delta_{y}$ and $\varepsilon_{y}$ are only determined by the $Y$-axis components.
} 
- $\quad$ common tension springs with non-zero free-length $L_{0}$ may be adopted [29];

- the range of suitable springs is widened, since actual spring dimensions must not fit the distance between the theoretical anchor points;

- cables and pulleys allow elastic elements to be arranged in more convenient locations, in particular near the mechanism base.

The CAD design of a practical solution for the balanced Orthoglide is presented in Fig. 4. Two tension springs, one for each balancing device, are mounted on the fixed base. The spring forces are transmitted to the theoretical anchor points on the slider and the platform by means of cables and pulleys. The red cable (and its corresponding pulleys) is used for the distal device and is connected to the moving platform; the blue cable (and its pulleys) is used for the proximal device and is attached to the vertical slider. The correct orientation of the final sections of cables between the theoretical anchor points is granted by the two pivoting pulleys. The balancing error introduced by the finite radius of the pivoting pulley on the distal device is expected to be negligible for practical purpose. However, if needed, it may be precisely cancelled adopting a pulley configuration similar to that proposed in [30], which exploits a second pulley installed at the cable anchor point. As for the distal device, one further pivoting pulley may be installed on the moving platform for replacing the fixed anchor point of the cable.

The presented solution allows the balancing springs to be mounted on the base rather than on the moving links. The springs undergo neither transverse motions nor rotations, thus significantly limiting potential elastodynamic issues. However the use cables and pulleys is known to possibly generate friction-related issues that should be carefully assessed in order to implement the device in practice.

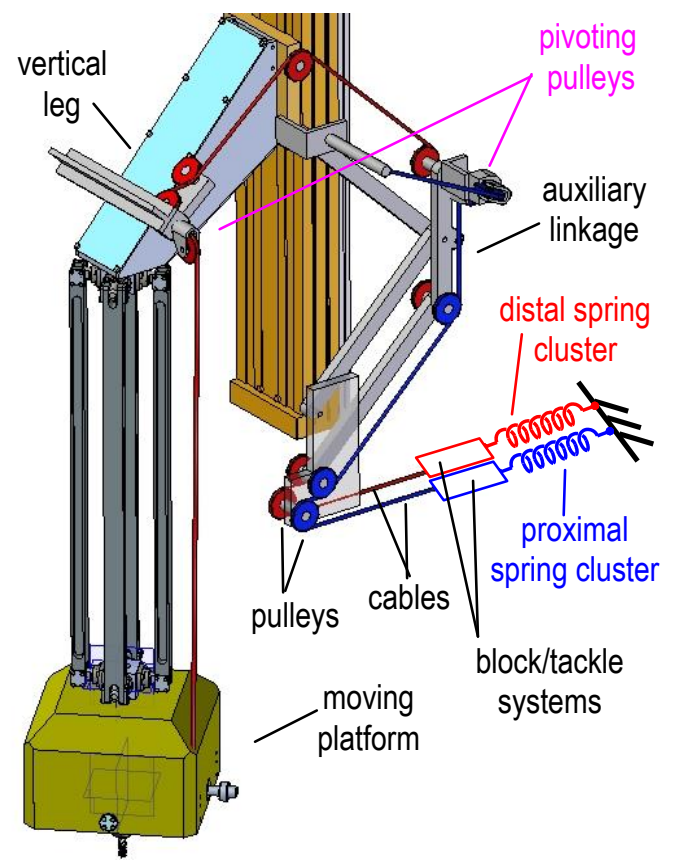

Fig. 4. Preliminary CAD representation of the balancing devices attached to the vertical leg. 
Table 1 Required balancing parameters.

\begin{tabular}{ccccccc}
\hline & \multicolumn{3}{c}{ distal device } & \multicolumn{3}{c}{ proximal device } \\
\cline { 2 - 7 } spring type & $\delta_{y}$ & $K_{D}$ & $f_{D, \max }$ & $\varepsilon_{y}$ & $K_{P}$ & $f_{P, \max }$ \\
& {$[\mathrm{mm}]$} & {$[\mathrm{N} / \mathrm{mm}]$} & {$[\mathrm{N}]$} & {$[\mathrm{mm}]$} & {$[\mathrm{N} / \mathrm{mm}]$} & {$[\mathrm{N}]$} \\
\hline theoretical & 150.00 & 2.510 & 2321 & 130.00 & 11.131 & 4470 \\
\hline off-the-shelf & 150.30 & 2.505 & 2318 & 131.52 & 11.002 & 4424 \\
\hline
\end{tabular}

In order to achieve a better distribution of loads, each balancing device may be doubled, thus installing two identical mechanisms working in parallel and arranged in a symmetric layout with respect to the vertical slider. Each one of the two symmetric mechanisms would be subject to half the loads, so that the force acting on each cable would also be halved. However, doubling cables and pulleys may also worsen possible issues concerning friction.

Different sets of parameters allowing the gravity compensation of the Orthoglide with the proposed balancing devices were examined. As described in Section 3.3, the spring anchor points can be arbitrarily set, thus obtaining the required spring rates, $K_{D}$ and $K_{p}$, from Eqs. (18) and (19). A suitable set of balancing parameters is shown in the first row of Table 1. For the sake of simplicity, the selected locations of the spring anchor points are reported only in terms of parameters $\delta_{y}$ and $\varepsilon_{y}$. Table 1 also reports the peak magnitude of the forces acting on the cables of the distal and proximal devices, $f_{D, \max }$ and $f_{P, \max }$ respectively, which is reached when the corresponding springs undergo their maximum elongations (thus depending on both the Orthoglide workspace specifications and the geometry chosen for the balancing devices). The values reported in the first row of Table 1 are referred to as theoretical since a further tuning is required to match the spring rates of commercial springs available in practice.

In order to meet the specifications of commercial springs, two expedients may be considered. On one hand, block and tackle systems (Fig. 5) may be used to, respectively, reduce by half and increase four times the spring elongation, $\Delta x_{s}$, and the spring stiffness, $K_{s}$, with respect to the values $\Delta x_{e q}$ and $K_{e q}$ available at the cables (which coincide with the theoretical values to be achieved). On the other hand, clusters of smaller tension springs working in parallel may be used in the place of single springs, with the additional advantage of reducing the influence of the statistical uncertainty that normally affects the specifications of commercial springs. A convenient combination of clusters of off-the-shelf springs and block-and-tackle systems is reported in Table 2. Parameters $K_{s}$ and $f_{s, \max }$ represent the spring rate and the maximum force of each spring of the cluster, respectively. The equivalent spring rates available at the cables with the chosen arrangement of off-the-shelf springs, as well as the adjusted locations of the spring anchor points, are reported in the second row of Table 1.

The selected set of balancing parameters allow potential interferences between the cables and the mechanism moving parts (in particular with the double parallelogram of the vertical leg) to be avoided. Moreover, it allows relatively large pulleys to be adopted, thus avoiding small-radius effects on cables. Finally, it entails a limited increment of the robot dimensions. 
Table 2 Parameters of a possible device implementation.

\begin{tabular}{cccccc}
\hline device & $\begin{array}{c}K_{s} \\
{[\mathrm{~N} / \mathrm{mm}]}\end{array}$ & $\begin{array}{c}f_{s, \max }[\mathrm{N}] \\
\text { distal }\end{array}$ & $\begin{array}{c}\text { \# springs } \\
\text { per cluster }\end{array}$ & $\begin{array}{c}\text { \# moving } \\
\text { pulleys }\end{array}$ & $\begin{array}{c}\text { total spring } \\
\text { mass }[\mathrm{Kg}]\end{array}$ \\
\hline proximal & 5.501159 & 8 & 2 & 8.087 \\
\hline
\end{tabular}

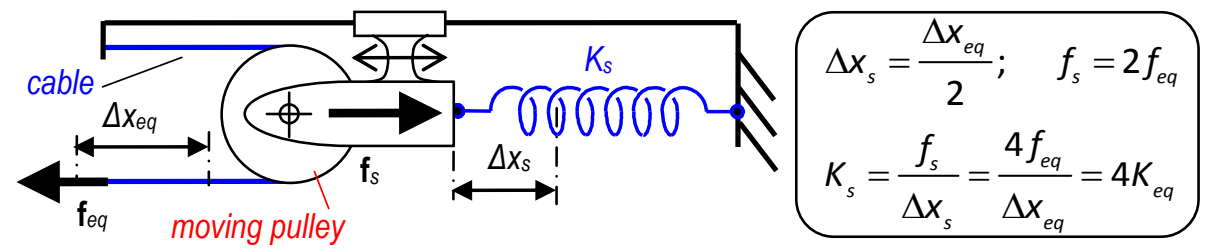

Fig. 5. Block and tackle solution applied to the proximal balancing device.

The proposed balancing devices do not require any actuator and completely compensate the static loads. They neither limit the mechanism workspace nor significantly modify the its overall dimensions, and they may be implemented with relatively simple modifications of the original system. Moreover, only small inertias are added, since the springs (which could be rather heavy) can be conveniently installed on the fixed base and no balancing counterweights are introduced, thus limiting the raise of dynamic loads $[20,21]$.

The presented solution for gravity compensation may be applied to other parallel mechanisms characterized by Linear-Delta architecture. Applying the method to manipulators featuring more than one leg with a translational axis parallel to gravity is straightforward. However, it is not difficult to extend this technique also to robots exhibiting legs with a generic orientation of the base-mounted prismatic joint. Details are omitted due to space limitations.

\section{DYNAMIC ANALYSIS}

The numerical models of the unbalanced Orthoglide and its balanced variant are implemented inside a multibody software environment (MSC ADAMS). The geometric and inertial properties are derived from the robot CAD model. In order to assess motor loads, a simplified kinematic chain, obtained by removing redundant constraints, is used. Simplified balancing devices are considered as well. Indeed, since the high cable stiffness and the low inertia of both cables and pulleys are expected to marginally influence the mechanism elastodynamic behavior, these parameters are not taken into account at this stage of the study. Accordingly, springs are modeled as massless and directly acting between the theoretical anchor points defined in Secs. 3.1 and 3.2

The dynamic operations of the unbalanced and the balanced robot are investigated by means of inverse-dynamics simulations, i.e. motion laws are imposed on the actuated prismatic joints, and the corresponding motor actions and joint reactions are computed.

\subsection{Simulated trajectories}

Two different groups of trajectories are tested. 
The first group, comprising Paths $A$ and $B$, aims at examining the mechanism behavior when the Orthoglide spans its workspace with velocities and accelerations in the upper range of its potentialities. Path $A$ prescribes point $P$ of the platform to follow, with constant velocity magnitude, a helix with radius $0.245 \mathrm{~m}$ and axis parallel to the $y-$ axis and passing through point $C$ (i.e. the center of the workspace). Two velocity profiles, $\# 1$ and \#2, are tested, corresponding to different values of the tangential velocity, respectively 0.8 and $1.2 \mathrm{~m} / \mathrm{s}$. The vertical velocity is set at $1 / 6$ of the tangential speed. Path $B$ is conceived to test both high speeds and frequent accelerations and decelerations. The platform moves along the edges and the diagonals of the workspace with a maximum acceleration of $1.5 \mathrm{~g}$ along each axis and two velocity profiles, $\# 1$ and $\# 2$, namely 0.6 and $1.0 \mathrm{~m} / \mathrm{s}$.

The second group of tests, comprising Paths $C$ through $F$, is conceived to simulate machining tasks. Four different cycles are simulated, namely rough-milling (Path $C$ ), finishing (Path D), deburring (Path E) and drilling (Path $F$ ), which are characterized by different dynamics and working loads. The first three cycles reproduce the machining of two rectangular pockets on horizontal planes, including rapid movements for tool positioning before and after each machining operation (characterized by maximum velocity and acceleration of, respectively, $1 \mathrm{~m} / \mathrm{s}$ and $1.5 \mathrm{~g}$ ). The fourth cycle simulates the execution of a sequence of holes, with rapid movements being performed before and after each drilling operation.

The tested velocities and accelerations (in particular relative to the rapid movements for tool positioning) are comparable to the maximum values characterizing the operation of commercially available machine tools with working specifications similar to the Orthoglide.

Cutting actions on the tool are modeled as forces proportional to the instantaneous platform velocity (i.e. the feed velocity). Their magnitudes are computed by using simplified relations normally adopted to estimate the spindle power requirements in aluminum machining operations [31]. For both roughing and finishing milling cycles, two equal-magnitude force components are taken into account, i.e. a tangential force orthogonal to both the feed direction and the vertical axis, and a radial force opposed to the feed velocity (which is a rather unfavorable case). An additional vertical force with the same magnitude of the other two is considered when deburring. In drilling simulations, only a thrust force opposite to the vertical feed is taken into account. The magnitudes of the feed velocity, $v_{f}$, and of the total cutting force, $F_{c}$, adopted in the simulations are provided in Table 3.

Table 3 Machining parameters adopted in simulations.

\begin{tabular}{lcr}
\hline \multirow{2}{*}{ Task } & \multicolumn{2}{c}{ Machining parameters } \\
\cline { 2 - 3 } & $v_{f}[\mathrm{~m} / \mathrm{s}]$ & $F_{c}[\mathrm{~N}]$ \\
\hline Path C: roughing & 0.10 & 1098 \\
\hline Path D: finishing & 0.10 & 101 \\
\hline Path E: deburring & 0.04 & 43 \\
\hline Path F: drilling & 0.04 & 500 \\
\hline
\end{tabular}




\subsection{Results}

The motor actions required to accomplish the two groups of operations are reported in Table 4 (Paths $A$ and $B$ ) and Table 5 (Paths $C$ through $F$ ), in terms of both rms and maximum absolute values, for the unbalanced (subscript "Un") and the staticallybalanced mechanism (subscript "SB"). The percentage variations induced by gravity compensation are expressed as:

$$
\Delta_{r m s \%}=100 \frac{r m s_{S B}-r m s_{U n}}{r m s_{U n}}, \quad \Delta_{\max \%}=100 \frac{\max _{S B}-\max _{U n}}{\max _{U n}}
$$

Since each motor acts along an axis of the global reference frame, motor forces are referred to by means of their corresponding axes.

Since the rms value provides an evaluation of the energy requirements of an actuator, it may also be adopted as a metric for a rough estimation of the global energy efficiency. To this purpose the overall rms of loads required for each tested trajectory (obtained by summing the rms values of the three motor loads) is computed and reported in both Table 4 and Table 5.

\subsubsection{Rapid positioning operations}

The motor actions required in the unbalanced robot are affected by both inertial and static loads. The contribution of static loads appears preponderant, since the force exerted by the vertical actuator is considerably greater than the horizontal actions and, due to the Orthoglide architecture, most of the gravity loads are sustained by the vertical leg. Consequently, the estimated overall energy requirements appear primarily ascribable to the vertical actuator. Indeed, the rms value of the force acting along the $y$ axis is about five times higher than the others in Path $A$, and three times higher in Path $B$, even in tests featuring greater inertia forces (velocity profile \#2).

Table 4 Computed motor actions for rapid positioning operations.

\begin{tabular}{|c|c|c|c|c|c|c|c|c|}
\hline \multirow[b]{2}{*}{ Path } & \multirow{2}{*}{$\begin{array}{l}\text { Vel. } \\
\text { profile }\end{array}$} & \multicolumn{7}{|c|}{ Dynamic motor loads [N] } \\
\hline & & axis & rmsun & rmSSB & $\Delta_{r m s} \%$ & $\max u_{n}$ & $\operatorname{maxsB}$ & $\Delta_{\max \%}$ \\
\hline \multirow{8}{*}{$A$} & \multirow{4}{*}{ \#1 } & $x$ & 56 & 96 & +72 & 150 & 190 & +26 \\
\hline & & y & 743 & 87 & -88 & 1887 & 1070 & -43 \\
\hline & & z & 83 & 99 & +19 & 264 & 405 & +53 \\
\hline & & tot & 882 & 283 & -68 & & & \\
\hline & \multirow{4}{*}{$\# 2$} & $x$ & 148 & 214 & +45 & 341 & 427 & +25 \\
\hline & & $\mathrm{y}$ & 743 & 114 & -85 & 1834 & 1016 & -45 \\
\hline & & z & 163 & 222 & +36 & 436 & 578 & +33 \\
\hline & & tot & 1054 & 550 & -48 & & & \\
\hline \multirow{8}{*}{$B$} & \multirow{4}{*}{ \#1 } & $x$ & 267 & 296 & +11 & 1189 & 1368 & +15 \\
\hline & & $\mathrm{y}$ & 843 & 342 & -59 & 2188 & 1565 & -28 \\
\hline & & $\mathrm{z}$ & 266 & 297 & +12 & 1189 & 1368 & +15 \\
\hline & & tot & 1375 & 935 & -32 & & & \\
\hline & \multirow{4}{*}{ \# 2} & $x$ & 309 & 347 & +12 & 1172 & 1347 & +15 \\
\hline & & $y$ & 861 & 401 & -53 & 2165 & 1537 & -29 \\
\hline & & $\mathrm{z}$ & 308 & 348 & +13 & 1172 & 1347 & +15 \\
\hline & & tot & 1477 & 1095 & -26 & & & \\
\hline
\end{tabular}


The statically balanced mechanism presents a remarkably less onerous load of the vertical actuator. In all cases, the rms of the vertical driving force is reduced by more than $50 \%$, and its maximum absolute value is reduced by roughly $1 / 3$. Conversely, an increment in the loads of both horizontal motors is observed for all simulated conditions, and it is particularly large for Path $A$. Nonetheless, the motor loads along the $x$ - and $z$ - axes remain relatively low, since they do not exceed $30 \%$ of the vertical force experienced in the unbalanced case, in terms of rms values. A reduction of the benefits brought about by gravity compensation is observed when the velocity grows, reasonably due to an increment in the inertial effects. Such a behavior is consistent with the results of other works (e.g. [13]). However also in the worst conditions, due to the remarkable decrease in the vertical motor force, static balancing results in a global reduction in the estimated energy requirements, as confirmed by the decrement of the overall rms.

\subsubsection{Machining operations}

The comparison between the motor loads of the unbalanced and the balanced mechanism, for all machining tasks, is reported in Table 5, as well as in Fig. 6 and Fig. 7, where the trends of the forces along the $y$ - and the z-axis are plotted as functions of time. Since the horizontal actuators behave similarly, the trend of the forces along the $x$ axis is not reported.

The vertical motor of the unbalanced mechanism still appears considerably affected by static loads (Fig. 6), with a high non-zero mean value being observed. However, inertial loads are also remarkable, in particular those associated with rapid movements for tool positioning. In addition, cutting forces cause significant oscillations of all motor loads.

Table 5 Computed motor actions for machining operations.

\begin{tabular}{lcrrrrrr}
\hline & \multicolumn{7}{c}{ Dynamic motor loads [N] } \\
\cline { 2 - 8 } Task & axis & rmsun & rmssB & $\Delta_{\text {rms } \%}$ & maxun & maxsB & $\Delta_{\text {max\% }}$ \\
\hline \multirow{3}{*}{ Path C: } & $\mathrm{X}$ & 569 & 574 & +1 & 1014 & 1142 & +13 \\
roughing & $\mathrm{y}$ & 813 & 326 & -60 & 1719 & 997 & -42 \\
& $\mathrm{z}$ & 765 & 758 & -1 & 1381 & 1216 & -12 \\
\cline { 2 - 8 } & tot & 2148 & 1659 & -23 & & & \\
\hline \multirow{3}{*}{ Path D: } & $\mathrm{X}$ & 134 & 131 & -2 & 863 & 858 & -1 \\
finishing & $\mathrm{y}$ & 777 & 159 & -80 & 1719 & 946 & -45 \\
& $\mathrm{z}$ & 197 & 157 & -21 & 867 & 861 & -1 \\
\cline { 2 - 8 } & tot & 1108 & 447 & -60 & & & \\
\hline \multirow{3}{*}{ Path E: } & $\mathrm{x}$ & 159 & 154 & -3 & 863 & 857 & -1 \\
deburring & $\mathrm{y}$ & 771 & 220 & -71 & 1744 & 973 & -44 \\
& $\mathrm{z}$ & 193 & 181 & -6 & 869 & 880 & +1 \\
\cline { 2 - 8 } & tot & 1123 & 555 & -51 & & & \\
\hline \multirow{3}{*}{ Path F: } & $\mathrm{x}$ & 206 & 208 & +1 & 1021 & 947 & -7 \\
drilling & $\mathrm{y}$ & 627 & 355 & -43 & 1768 & 1029 & -42 \\
& $\mathrm{z}$ & 136 & 139 & +2 & 977 & 940 & -4 \\
\hline & tot & 970 & 702 & -28 & & & \\
\hline
\end{tabular}



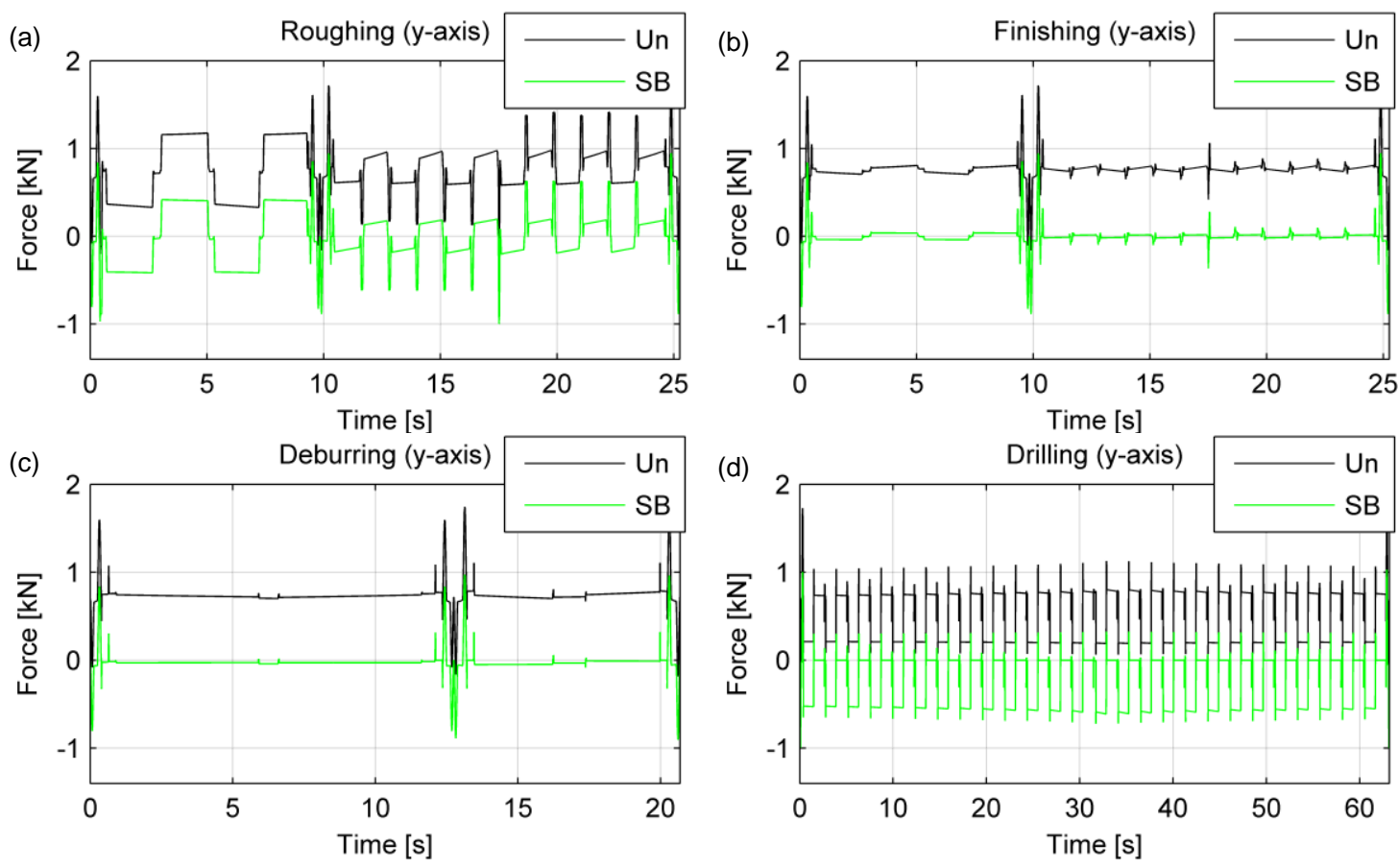

Fig. 6. Machining operations, comparison between motor loads of the unbalanced (Un) and the balanced (SB) robot along the $y$-axis
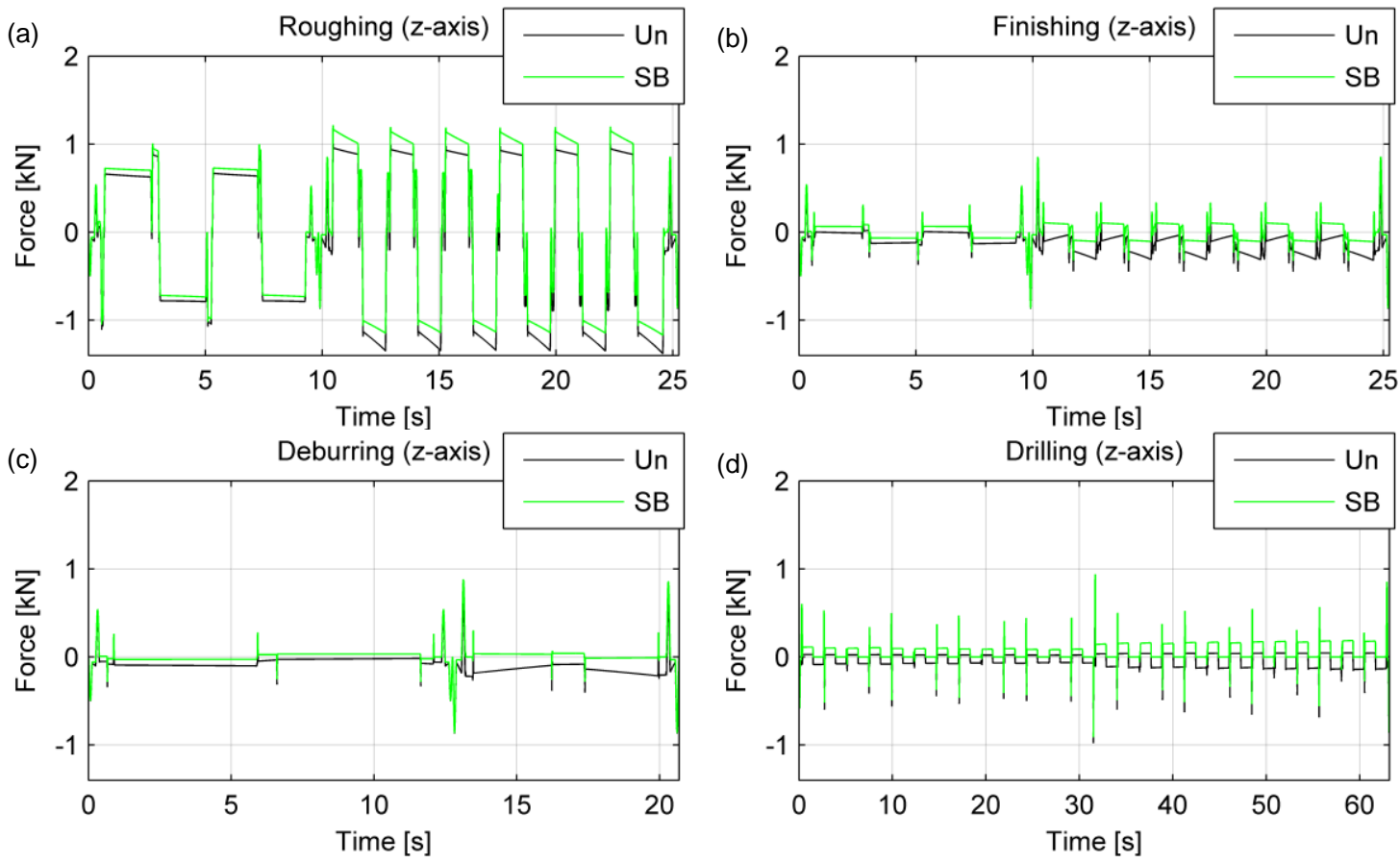

Fig. 7. Machining operations, comparison between motor loads of the unbalanced $(U n)$ and the balanced $(S B)$ robot along the $z$-axis. 
In all machining operations, gravity compensation significantly decreases the vertical motor loads with respect to the unbalanced system, in terms of both rms and maximum absolute values. Conversely the forces of the horizontal actuators are only marginally affected by compensation, and both their rms and maximum absolute values remain essentially unvaried.

\subsubsection{Discussion}

It emerges from Secs. 5.2.1 and 5.2.2 that the overall rms value of motor loads appear significantly reduced by gravity compensation for all tested working conditions, in both rapid positioning operations and typical machining tasks. Since such parameter is used for approximately estimating the energy requirements over the working cycles, the simulations results confirm that an appreciable increment in energy efficiency should be reasonably expected, at least for the tested functioning conditions.

The numerical simulations do not reveal any critical drawback possibly associated with the balancing device, even if a further assessment of potential elatodynamic or friction-related issues may be advisable.

Even if analyses were performed over specific imposed motions, the simulated conditions are fairly representative of the majority of the working cycles that may characterize the Orthoglide operation, including the most onerous tasks. Hence, the results drawn from simulation are believed to possess a wide validity for the robot at hand, showing that static balancing may be a viable strategy for enhancing the Orthoglide working performance.

\section{SENSITIVITY ANALYSIS}

A sensitivity analysis is carried out in order to assess the robustness of the proposed gravity-compensation approach, with respect to possible inaccuracies affecting the parameters of the balancing devices. The variations of the following parameters are taken into account:

1. mass of the moving platform, $m_{p}$, which may change due to the installation of different cutting tools;

2. mass of the vertical translating slider, $m_{s 2}$, which may be inaccurately estimated due to partial data available for commercial actuators;

3. stiffness, $K_{r}$ and $K_{t}$, of the tension springs used in the distal and proximal balancing devices (discrepancies between actual and nominal values can be normally found in off-the-shelf springs);

4. location of the cable anchor point on the platform, $\mathbf{r}_{B}=\left(r_{B x}, r_{B y}, r_{B z}\right)$, possibly affected by manufacturing or assembly inaccuracies.

The effects induced by modifications of these parameters with respect to their nominal values are investigated by means of static simulations. The parameters 1-3 of the balanced model are altered by $\pm 10 \%$ of their rated value, whereas an absolute deviation of $\pm 5 \mathrm{~mm}$ is imposed on each component of the parameter 4 . For each value assigned to a single parameter, the platform is driven across the complete workspace in static conditions, i.e. all inertial actions are neglected. The residual motor loads (i.e. the motor actions required for maintaining neutral equilibrium after compensation) are 
Table 6 Results of the sensitivity analysis.

\begin{tabular}{|c|c|c|c|c|c|c|c|c|c|c|c|c|c|c|c|c|c|c|c|c|c|c|}
\hline \multicolumn{23}{|c|}{ Static motor loads [N] } \\
\hline \multirow{2}{*}{\multicolumn{3}{|c|}{ unbalanced }} & \multicolumn{20}{|c|}{ statically balanced } \\
\hline & & & \multicolumn{4}{|c|}{ case $1\left(m_{p}\right)$} & \multicolumn{4}{|c|}{ case $2\left(m_{s 2}\right)$} & \multicolumn{4}{|c|}{ case $3\left(K_{r}\right)$} & \multicolumn{4}{|c|}{ case $4\left(K_{t}\right)$} & \multicolumn{4}{|c|}{ case $5\left(r_{B}\right)$} \\
\hline axis & rms & $\max$ & rms & $\Delta_{m s} \%$ & $\max$ & $\Delta_{\max \%}$ & rms & $\Delta_{m s} \%$ & $\max$ & $\Delta_{\max \%}$ & $\mathrm{rms}$ & $\Delta_{m s} \%$ & $\max \angle$ & $\Delta_{\max \%}$ & $\mathrm{rms}$ & $\Delta_{m m s} \%$ & $\max$ & $\Delta_{\max \%}$ & $\mathrm{rms}$ & $\Delta_{m s} \%$ & $\max \angle$ & $\Delta_{\max \%}$ \\
\hline$x$ & 88 & 243 & 9 & -90 & 24 & -90 & 0 & -100 & 0 & -100 & 3 & -97 & 8 & -97 & 0 & -100 & 0 & -100 & 16 & -82 & 31 & -87 \\
\hline$y$ & 728 & 907 & 3 & -100 & 19 & -98 & 72 & -90 & 72 & -92 & 13 & -98 & 19 & -98 & 27 & -96 & 27 & -97 & 7 & $7 \quad-99$ & 19 & -98 \\
\hline$z$ & 88 & 243 & 9 & -90 & 24 & -90 & 0 & -100 & 0 & -100 & 3 & -97 & 8 & -97 & 0 & -100 & 0 & -100 & 16 & $6-82$ & 31 & -87 \\
\hline
\end{tabular}

monitored in a grid of reference points. The rms and maximum absolute values are computed and compared with the values obtained from static simulations of the unbalanced model (performed by considering the same positions in the workspace and without modifying the model parameters). The simulation results are summarized in Table 6.

Since identical absolute values are obtained for both the positive and the negative variations of each parameter, results are shown only for positive variations. Percentage variations $\Delta_{r m s}$ and $\Delta_{m a x}$ of the driving-force rms and maximum absolute values with respect to the unbalanced case are also shown.

Static loads of the unbalanced mechanism are dominated by the vertical component. Indeed, due to the system architecture, the weight of all moving parts is almost completely sustained by the vertical actuator.

In the balanced mechanism, static loads are significantly lowered, in spite of the modified parameters, with the reduction being about $90 \%$ in both the rms and the maximum absolute values.

Based on these results, gravity compensation appears to be effective within the entire range of the analyzed parameters, thus the robustness of the proposed design proving satisfactory.

\section{CONCLUSIONS}

This paper studied the static balancing of the Orthoglide 5-axis, a prototypal PKM for machining applications that features a Linear-Delta architecture. Gravity compensation was achieved by attaching tension springs and a simple auxiliary linkage to the robot vertical leg. The mechanical design of a solution for implementing balancing in practice was presented. A suitable arrangement of cables and pulleys was defined to apply the spring forces. The main features of the proposed design are the following:

- only relatively simple modifications of the original robot are needed in order to properly install the balancing devices, with negligible increment of the overall robot dimensions;

- the effectiveness of the balancing devices is weakly influenced by possible inaccuracies affecting the design parameters (as proven by a sensitivity analysis), thus allowing design tolerances to be relieved;

- the use of cables and pulleys allows the balancing springs to be installed on the fixed base, thus limiting both the additional inertia on the moving parts and the motion of the springs (which may lead to elastodynamic issues); 
- $\quad$ the proposed devices operate passively, i.e. they do not require additional actuators that would entail additional power consumption.

The results provided by numerical simulations of the Orthoglide operation in typical working conditions showed that:

- the introduction of the balancing devices causes only a small increment in the moving inertias, which do not significantly affect the robot dynamic performance;

- a significant reduction in the overall motor loads, as well as a more uniform loading of the actuators, is achieved when the robot performs common machining tasks.

Based on the results presented in this work, gravity compensation appears as a viable strategy for enhancing the operation of the Orthoglide and, to a certain extent, of PKMs for machining applications characterized by similar functioning conditions.

The proposed balancing approach, although developed for the specific case of the Orthoglide 5-axis, may be extended to other machines featuring a Linear-Delta architecture.

\section{ACKNOWLEDGMENT}

The authors wish to thank Prof. Philippe Wenger, Dr. Stephane Caro and Dr. Damien Chablat from the IRCCyN institute of Nantes, France, for providing information and data concerning the Orthoglide 5-axis.

\section{REFERENCES}

[1] S.K. Agrawal, A. Fattah, Gravity-balancing of spatial robotic manipulators, Mech. Mach. Theory 39 (12) (2004) 1331-1344.

[2] V. Arakelian, S. Ghazaryan, Improvement of balancing accuracy of robotic systems: Application to leg orthosis for rehabilitation devices, Mech. Mach. Theory 43 (5) (2008) 565-575.

[3] Y.-Y. Lee, D.-Z. Chen, Determination of spring installation configuration on statically balanced planar articulated manipulators, Mech. Mach. Theory 74 (2014) 319-336.

[4] C. Cho, S. Kang, Design of a static balancing mechanism with unit gravity compensators, Proc. of the 2011 IEEE/RSJ International Conference on Intelligent Robots and Systems, San Francisco, CA, USA, 2011, pp. 1857-1862.

[5] T. Morita, F. Kuribara, Y. Shiozawa, S. Sugano, A novel mechanism design for gravity compensation in three dimensional space, Proc. Of the 2003 IEEE/ASME International Conference on Advanced Intelligent Mechatronics, Kobe, Japan, 2003, pp. 163-168.

[6] D.A. Streit, E. Shin, Equilibrators for planar linkages, ASME J. Mech. Des. 115 (3) (1993) 604-611.

[7] T. Wongratanaphisan, M.O.T. Cole, Analysis of a Gravity Compensated Four-Bar Linkage Mechanism With Linear Spring Suspension, ASME J. Mech. Des., 130 (1):011006 (2008) 1-8.

[8] S.R. Deepak, G.K. Ananthasuresh, Static balancing of a four-bar linkage and its cognates, Mech. Mach. Theory 48 (2012) 62-80. 
[9] T. Laliberté, C.M. Gosselin, Static Balancing of 3-DOF Planar Parallel Mechanisms, IEEE-ASME Trans. Mechatron. 4 (4) (1999) 363-377.

[10]J. Wang, C.M. Gosselin, Static balancing of spatial four-degree-of-freedom parallel mechanisms, Mech. Mach. Theory 35 (4) (2000) 563-592.

[11]C.M. Gosselin, J. Wang, Static balancing of spatial six degree-of-freedom parallel mechanisms with revolute actuators, J. Robotic Syst. 17 (3) (2000) 159-170.

[12]A. Russo, R. Sinatra, F. Xi, Static balancing of parallel robots, Mech. Mach. Theory 40 (2) (2005) 191-202.

[13]M. Carricato, C.M. Gosselin, A Statically Balanced Gough/Stewart-Type Platform: Conception, Design, and Simulation, ASME J. Mech. Robot. 1 (3):031005 (2009) 116.

[14]I. Ebert-Uphoff, C.M. Gosselin, T. Laliberté, Static balancing of spatial parallel platform mechanisms-revisited, ASME J. Mech. Des. 122 (1) (2000) 43-51.

[15]C. Baradat, V. Arakelian, S. Briot, S. Guegan, Design and prototyping of a new balancing mechanism for spatial parallel manipulators, ASME J. Mech. Des. 130 (7):072305 (2008) 1-13.

[16]S. Perreault, P. Cardou, C.M. Gosselin, Approximate static balancing of a planar parallel cable-driven mechanism based on four-bar linkages and springs, Mech. Mach. Theory 79 (2014) 64-79.

[17]G.G. Lowen, F.R. Tepper, R.S. Berkof, Balancing of linkages-an update, Mech. Mach. Theory 18 (3) (1983) 213-220.

[18]E. Raghu, A. Balasubramonian, Experimental study on the elastodynamic behavior of the unbalanced and the counterweighted four bar mechanisms, ASME J. Mech. Des. 112 (3) (1990) 271-277.

[19]M.J. Walker, R.S. Haines, An experimental study of the effects of counterweights on a six-bar chain, Mech. Mach. Theory 17 (6) (1982) 355-360.

[20]A. Martini, M. Troncossi, M. Carricato, A. Rivola, Elastodynamic behaviour of balanced closed-loop mechanisms: numerical analysis of a four-bar linkage, Meccanica 49 (3) (2014) 601-614.

[21]A. Martini, M. Troncossi, A. Rivola, Elastodynamic effects of mass-balancing: experimental investigation of a four-bar linkage, Adv. Mech. Eng. DOI: 10.1155/2013/949457, (2013) 1-10.

[22]Y. Li, J. Wang, X.-J. Liu, L.-P. Wang, Dynamic performance comparison and counterweight optimization of two 3-DOF parallel manipulators for a new hybrid machine tool, Mech. Mach. Theory 45 (11) (2010) 1668-1680.

[23]G. Stan, R. Ciobanu, A. Pal, Balancing-compensation system for the vertically moving elements of the machine tools with numerical control, Meccanica 46 (4) (2011) 755769.

[24]S. Lessard, P. Bigras, I.A. Bonev, A New Medical Parallel Robot and Its Static Balancing Optimization, ASME J. Med. Devices 1 (4) (2007) 272-278.

[25]M.B. Dehkordi, A. Frisoli, E. Sotgiu, M. Bergamasco, Modelling and Experimental Evaluation of a Static Balancing Technique for a new Horizontally Mounted 3-UPU Parallel Mechanism, Int. J. of Adv. Robot. Syst. 9 (193) (2012) 1-12. 
[26]R. Ur-Rehman, S. Caro, D. Chablat, P. Wenger, Kinematic and dynamic analyses of the Orthoglide 5-axis, Proc. of the 7th France-Japan, 5th Europe-Asia Congress on Mechatronics, Le Grand Bornand, France, 2008.

[27]R.H. Nathan, A constant force generation mechanism, ASME J. Mech. Transm. Autom. Des. 107 (4) (1985) 508-512.

[28]A. Pashkevich, D. Chablat, P. Wenger, Kinematics and workspace analysis of a threeaxis parallel manipulator: the Orthoglide, Robotica 24 (1) (2006) 39-49.

[29]J.L. Herder, Energy-free systems: theory, conception and design of statically balanced spring mechanisms, Ph.D. thesis, Delft University of Technology, Netherlands (2001).

[30]R. Barents, M. Schenk, W.D. van Dorsser, B.M. Wisse, J.L. Herder, Spring-to-spring balancing as energy-free adjustment method in gravity equilibrators, ASME J. Mech. Des. 133 (6):061010 (2011) 1-10.

[31]Tungaloy cutting tools general catalogue, Chapter 15: Technical reference. 